\title{
The Potential Use of Intrauterine Insemination as a Basic Option for Infertility: A Review for Technology-Limited Medical Settings
}

\author{
Abdelrahman M. Abdelkader and John Yeh \\ Department of Gynecology-Obstetrics, University at Buffalo, The State University of New York, Buffalo, NY 14222, USA \\ Correspondence should be addressed to John Yeh, jyeh@buffalo.edu \\ Received 5 November 2008; Accepted 15 January 2009 \\ Recommended by Faustino R. Pérez-López
}

Objective. There is an asymmetric allocation of technology and other resources for infertility services. Intrauterine insemination (IUI) is a process of placing washed spermatozoa transcervically into the uterine cavity for treatment of infertility. This is a review of literature for the potential use of IUI as a basic infertility treatment in technology-limited settings. Study design. Review of articles on treatment of infertility using IUI. Results. Aspects regarding the use of IUI are reviewed, including ovarian stimulation, semen parameters associated with good outcomes, methods of sperm preparation, timing of IUI, and number of inseminations. Implications of the finding in light of the needs of low-technology medical settings are summarized. Conclusion. The reviewed evidence suggests that IUI is less expensive, less invasive, and comparably effective for selected patients as a first-line treatment for couples with unexplained or male factor infertility. Those couples may be offered three to six IUI cycles in technology-limited settings.

Copyright (C) 2009 A. M. Abdelkader and J. Yeh. This is an open access article distributed under the Creative Commons Attribution License, which permits unrestricted use, distribution, and reproduction in any medium, provided the original work is properly cited.

\section{Introduction}

Intrauterine insemination (IUI) is the therapeutic process of placing washed spermatozoa transcervically into the uterine cavity for the treatment of infertility. IUI theoretically allows a relatively higher number of motile spermatozoa to reach the oocyte [1]. The rationale for washing sperm is to remove prostaglandins, infectious agents, and antigenic proteins as well as to remove immotile spermatozoa, leucocytes, and immature germ cells. The process allows the concentration of spermatozoa in a small volume of culture media and then the concentrated spermatozoa is placed into the uterus through a transcervical catheter.

The general process of artificial insemination has been used to treat a variety of physiological and psychological male and female infertility disorders such as severe hypospadius, retrograde ejaculation, impotence, and vaginismus. IUI, in the past, has been used as treatment for poor postcoital tests and immunologic infertility [2]. Currently, IUI is used to treat moderate male factor infertility and unexplained infertility. Another common use of IUI is to enhance the efficacy of treatment by ovulation induction for ovulatory disorders [1]. The simple and noninvasive nature of IUI has allowed it to be performed by nurses in some centers with analogous pregnancy rates to the procedures performed by physicians [3]. Factors that may influence IUI outcome include the use of ovulation induction agents, semen analysis parameters, techniques used for sperm preparation, and the timing and number of inseminations.

The resource allocation for advanced infertility services is asymmetric, with the expertise and technology more concentrated in the larger cities and resource-rich westernized countries [4]. In this review, we address the clinical situation in the majority of the occasions, in which the infertility technology that is available for treatment of patients may be limited. Outside the technologically advanced centers, the majority of the infertility patients have fewer options for treatment. The roles for IUI and considerations for its advantageous use in this limited technology setting are considered in this manuscript. This review is divided into two parts. In the first, the efficacy and cost-effectiveness of IUI are discussed, and, in the second part, the evidence is presented for the beneficial clinical practice of IUI in limited technology settings. 


\section{Infertility and Its Economic and Psychological Impacts}

Infertility is defined as failure to achieve pregnancy for one year or more without use of contraception during the childbearing period and affects approximately 10-15\% of couples [5]. In 1995, The National Survey of Family Growth reported that 9.3 million women between 1544 years (15\% of women of reproductive age) made use of infertility services in the United States. These services included medical advice, testing for both couples, drugs for ovulation induction, corrective surgery for tubal blockage, and assisted reproductive technologies (ARTs) [6]. The costs of infertility evaluation and therapy were evaluated in a study conducted by Stovall et al. [7]. They found that the costs of infertility services ranged from 0.36 to $1.03 \%$ of a total health care plan with an average of $0.8 \%$. In a 3-year period, they found that infertility cost was $\$ 680921$ of $\$ 86445642$ total health care plan costs.

In addition to its economic costs, infertility has a major psychological impact. Oddens et al. reported that infertile women had depressive and anxiety symptoms four times more frequently than fertile women [8], and the rate is comparable to women with cancer and coronary heart disease [9]. The presence of such psychological disorders may have a negative impact on infertility treatment outcomes, as it was reported that IVF success rates in the first 5 cycles may be lower in depressed women compared to the nondepressed [10].

\section{Limitations of Assisted Reproductive Technologies}

In 2001 in the United States, 108130 ART cycles were carried out in 385 programs, with 79042 cycles being IVF [11]. In 2003 in Europe, 365103 ART cycles were accomplished in 1008 clinics, with 132932 cycles being IVF [12]. Despite this increase in the use of these treatment modalities, they are still costly, invasive, and associated with drawbacks. In the year 2001, the median cost of IVF in the US was extrapolated to be $\$ 9226$ per cycle and $\$ 56419$ per delivery. Outside the US, this cost was calculated to be $\$ 3531$ per cycle and $\$ 20522$ per delivery [13]. The high cost of IVF deepens on the gap between the number of IVF cycles performed and the treatment needs of infertile couples.

In addition to its costs, the use of IVF is associated with other undesirable outcomes. These include preterm delivery, leading to increased risk of newborn prematurity and its concomitant costs and morbidity, and increased rates of multiple gestations [14]. In the US, in the year 2003, 48756 infants were born from ARTs, accounting for $1 \%$ of all births. Fifty-one percent of this number resulted from multiple gestations. Furthermore, the incidence of low birth weight and preterm delivery among singleton infants born from ARTs were $9 \%$ and $15 \%$, respectively, while low birth weight occurred in $94 \%$ among triplets and higher order multiple gestations [15]. In a meta-analysis to assess the perinatal outcome in singletons following IVF [16], Jackson et al. reported increased fetal risks of preterm birth, low and very low birth weights, small for gestational age, neonatal intensive care unit admissions, stillbirth, neonatal mortality, and cerebral palsy as well as increased maternal risks of pre-eclampsia, placenta previa, gestational diabetes, and caesarean delivery. Furthermore, there may be an increased incidence of major birth defects (renal and musculoskeletal) and autosomal reciprocal-balanced translocations among infants born after IVF/ICSI compared to naturally conceived infants $[17,18]$.

\section{Limitations of IUI}

The limitations of IUI are few. They included the potential risk of infection from uterine catheterization and injection of the semen specimen. However, this risk has been reported to be $0.01-0.2 \%$. In addition, ectopic pregnancies and spontaneous miscarriages may occur, which is not different from other infertility treatment modalities [19]. The production of antisperm antibodies is another hypothetical drawback of the use of IUI. The incidence of multiple pregnancy and ovarian hyperstimulation syndrome (OHSS) with IUI may occur and is related to the use of ovarian stimulation with IUI. The risk of OHSS may be decreased by use of low-dose drug regimens, close monitoring, and strict cycle cancellation criteria.

Regarding the perinatal outcomes of IUI conceptions, Gaudoin et al. reported in a retrospective cohort study that ovulation induction combined with IUI was associated with increased risk of preterm birth and low birth weight [20]. However, other studies did not describe such associations $[21,22]$.

\section{Effectiveness of IUI}

The pregnancy rate of IUI is reported to $10-20 \%$ per patient, but the reported rates range from as low as $5 \%$ to as high as $70 \%$ [23]. Based on the etiology of infertility, the highest rates were reported when IUI was used in patients with anovulation who were undergoing ovulation induction therapy at the time of the IUI treatment, male factor infertility, and unexplained infertility. In patients with endometriosis, the pregnancy rates were the lowest [24]. The number of mature follicles $(17 \mathrm{~mm}$ in diameter or more) is another prognostic factor in IUI success, where the presence of 3-4 mature follicles was associated with higher pregnancy rates and a lower incidence of high-order multiple pregnancies [25]. Other prognostic factors included female age, infertility duration and amount of motile sperm [26].

The cost effectiveness of IUI has been studied. In a retrospective cohort study [27], Van Voorhis et al. reported $\$ 8674$ as the cost per delivery for IUI alone, $\$ 7808$ for clomiphene combined with IUI, \$10282 for human menopausal gonadotropin (hMG) combined with IUI and $\$ 43138$ for IVF. The authors supported the use of IUI (any modality) as being cost effective, before ART, for infertile couples with patent tubes, female age 38 years or less, and a sperm count of $10 \times 10^{6}$ motile spermatozoa in a post wash 
sample. For those with blocked tubes, IVF-ET was found to be a cost-effective practice compared to surgery. In a prospective study, based on cost effectiveness, Karande et al. did not recommend IVF as first-line option for infertile couples [28]. In a prospective-randomized study, Goverde et al. found that IUI was as effective as and less costly than IVF in treatment of unexplained and male factor infertility [29]. Peterson et al. reported that four cycles of controlled ovarian hyperstimulation $(\mathrm{COH})$ combined with IUI were superior to IVF and less expensive than single IVF cycle [30]. Even when the same stimulation protocols were used, the cost per pregnancy for IUI was less than half that of IVF [31]. Cohlen et al. conducted a Cochrane review to analyze the effectiveness of IUI versus timed intercourse both in natural and stimulated cycles in cases of male factor infertility [32]. A search of Medline (January 1966-present), EMBASE (http://www.embase.com/), DDFU (http://www.ovid.com/site/catalog/DataBase/893.jsp?top = 2 \&mid $=3 \&$ bottom $=7 \&$ subsection=10), BIOSIS (Philadelphia, USA), SCI (http://www.thomsonreuters.com/products_services/scientific/Science_Citation_Index_Expanded) and hand searching of references from identified studies resulted in 43 studies related to research topic being retrieved. They used 17 studies in the systematic review and their data were pooled in the meta-analysis. In their final conclusions, Cohlen et al. reported that IUI with $\mathrm{COH}$ was more costeffective compared to IVF [32]. A more recent study, by Pashayan et al., however, offered evidence that IVF as a first-line treatment for couples with unexplained and mild male factor infertility was less costly than IUI followed by IVF (for IUI treatment inability to achieve pregnancy) [33].

\section{The Use of IUI and Concurrent Ovarian Stimulation}

Which is more effective: IUI in a natural cycle or combined with ovarian stimulation? In a meta-analysis that included only randomized controlled trials, Hughes reported a significant improvement in fecundity with the use of IUI and follicle stimulating hormone (FSH). He found more than two-fold increase in fecundability for either IUI or FSH treatment alone, while he found a five-fold increase when IUI and FSH are combined together [34]. In the Cochrane review by Cohlen et al. referenced above, conducted to assess the effectiveness of IUI versus timed intercourse [32], IUI in a natural cycle was compared to IUI combined with controlled ovarian hyperstimulation. Data from four trials were included in the meta-analysis for this comparison. Although there was no significant difference between IUI in natural cycles and IUI combined with ovarian hyperstimulation in cases with male factor infertility, the results suggested increased conception with ovarian hyperstimulation (odds ratio: 1.8 with CI: $0.98-$ 3.3). In their conclusion, Cohlen et al. recommended the use of IUI in natural cycles for cases with severe semen defect (a total motile sperm count of less than $10 \times$ $10^{6}$, but with more than $1 \times 10^{6}$ motile sperm after preparation). In cases of less severe semen defect, IUI with ovarian hyperstimulation was recommended. A large randomized study in the US confirmed the effectiveness of combining ovarian stimulation with IUI [35]. The study reported an overall pregnancy rate per couple of 33\% for IUI with ovarian stimulation and an overall pregnancy rate of $18 \%$ for IUI alone. Data of this study were included in the Cochrane review, discussed above, which was accomplished to assess the effectiveness of IUI in the treatment of unexplained infertility. Verhulst et al. conducted a Cochrane review to assess the effectiveness of IUI in the treatment of unexplained infertility [36]. Their primary search yielded 198 articles related to the topic, but hand searching of their abstracts resulted in 25 trials included in the review. It was found that live birth rate per couple was significantly higher when IUI was combined with ovarian hyperstimulation compared to IUI alone (OR 2.07, 95\% CI: 1.22-3.5) [36]. However, this review did not find studies assessing expectant management versus IUI in unexplained infertility. At the same year of publication as the above Cochrane review, Steures et al. published a randomized clinical trial comparing ovarian hyperstimulation combined with IUI versus expectant management for couples with unexplained infertility [37]. This study reported no higher pregnancy rates for ovarian hyperstimulation combined with IUI overexpectant management in couples with unexplained infertility.

The National Institute for Clinical Excellence (NICE) in the United Kingdom recommended, with level I evidence, that when IUI is used to treat male factor infertility, ovarian stimulation should not be offered. This conclusion was based on two trials $[29,32]$. Supporting this recommendation is the results of a randomized clinical trial conducted in the Netherlands by Steures et al. [38]. This study reported similar pregnancy rates when IUI alone or IUI combined with ovarian hyperstimulation were used to treat couples with abnormal postcoital tests and poor prognosis for spontaneous pregnancy. For unexplained infertility, both IUI alone and IUI combined with ovarian hyperstimulation appear to be more effective than expectant management alone [39].

What drugs should be used for ovarian hyperstimulation combined with IUI? Gonadotropins with and without gonadotropin-releasing hormone $(\mathrm{GnRH})$ agonist/antagonist have been studied, along with recombinant human FSH ( $\mathrm{rFSH})$. Ragni et al. reported that the daily use of $50 \mathrm{IU}$ of $\mathrm{rFSH}$, combined with a GnRH antagonist, ganirelix, for ovarian stimulation prior to IUI resulted in a live birth rate per couple of $25.7 \%$ [40]. When the same dose of $\mathrm{rFSH}$ was used on alternate days, $\mathrm{rFSH}$ produced a live birth rate of $2.9 \%$ [40]. In another study, 50 IU daily of $\mathrm{rFSH}$ was found similar to 75 IU daily of urinary FSH (uFSH) in the outcomes of the number of follicles $>17 \mathrm{~mm}$ and days of stimulation [41]. The clinical pregnancy rates were $12.7 \%$ for rFSH and $11.9 \%$ for uFSH [41]. Thus, the more expensive rFSH was not a cost-effective drug for induction of ovulation in IUI cycles. Conversely, Demirol and Gurgan compared rFSH to uFSH and hMG in IUI cycles, and found rFSH and IUI produced a clinical pregnancy rate of $25.9 \%$ compared to $13.8 \%$ for uFSH and $12.5 \%$ for hMG [42]. When rFSH 
and clomiphene citrate (CC) were compared for ovulation induction with IUI in couples with unexplained and male factor infertility, the cumulative pregnancy rate was $38 \%$ for CC-IUI group versus $34.3 \%$ for the rFSH-IUI group [43].

Recently Cantineau and Cohlen performed a Cochrane review to evaluate different ovarian stimulation protocols (antiestrogens, aromatase inhibitors, and gonadotropins with or without GnRH agonists/antagonists) [44]. All published randomized controlled trials (RCTs) comparing different stimulation protocols before IUI were searched in the Menstrual Disorders and Subfertility group's Central register of the Controlled Trials, Medline, and EMBASE. 81 studies were identified, but only 43 trials met the inclusion criteria. Of the different comparisons done in this review, letrozole was not more effective than CC (OR 1.2, 95\% CI: 0.64-2.1). The analysis also revealed that gonadotropins, in low-dose regimens (50-75 IU), were the most effective agents when ovarian stimulation was combined with IUI. Although less effective than gonadotropins, antiestrogens were more cost effective in IUI therapy. Neither a higher dosage ( $>75$ IU gonadotropins) nor the addition of GnRH agonists was more effective. Conversely, the higher dosage and the $\mathrm{GnRH}$ agonists were associated with increased costs and risks of multiple gestations and of OHSS. A systematic review by Costello assessed the effectiveness of CC and IUI in the treatment of ovulatory infertility [45]. Seven RCTs were included in this review. The meta-analysis of this review showed a cycle pregnancy rate of $14.3 \%$ for CC combined with IUI versus $6.4 \%$ for natural cycle IUI.

Considerations in Technology-Limited Settings. The evidence reviewed suggests that oral clomiphene therapy combined with IUI or natural cycle IUI is satisfactory first-line choices for treatment of unexplained and male infertility in low-technology settings. The combination of IUI and controlled ovarian hyperstimulation may result in a relatively higher therapeutic pregnancy rate for unexplained and for male infertility. Gonadotropins are the controlled ovarian hyperstimulation agents that result in the highest rates of pregnancy. However, the costs associated with these drugs are higher, and the serum steroid measurements and ultrasonographic monitoring require higher levels of technological capabilities.

\section{Semen Parameters and the Use of IUI}

The WHO criteria for normal semen parameters are widely used [46]. In the system, an abnormal semen count is defined as deviations from the normal criteria on two consecutive semen analyses [46]. Studies have examined semen parameters, like motile sperm concentration and normal sperm morphology, in relation to IUI outcome (Table 1). In Table 1, four inferences could be made: most of the studies are retrospective, the number of insemination cycles is fewer in the prospective studies, all but one study used the inseminated motile sperm count after sperm preparation and the studies reported on different outcome measurements. In spite of the limitations outlined above, it may be deduced that reasonable IUI success rates can still be obtained in cases of severe semen defects (normal morphology $4 \%$ or less and inseminated sperm count as low as one million). This supports the need for large well-designed prospective studies using standard outcomes.

Regarding sperm concentration, Ombelet et al. in a retrospective study found that when inseminated motile sperm count was $>1 \times 10^{6}$, clomiphene-IUI resulted in a baby take-home rate of $21-25 \%$ after 3 cycles [47]. Even when inseminated motile sperm count was $<1 \times 10^{6}$ and the normal sperm morphology $>4 \%$, clomiphene-IUI remained a treatment choice that resulted in pregnancies. In a retrospective analysis of 3479 IUI cycles conducted for 1039 infertile couples [48], Van Voorhis et al. found that a total motile sperm count per specimen of less than 10 million was associated with lower pregnancy rates. On the other hand, a total motile sperm count above 10 million did not produce a significant increase in IUI pregnancy rates.

Cohlen et al. defined a total motile sperm count per ejaculate between 5 and $10 \times 10^{6}$ to be a severe semen defect [49]. They reported a pregnancy rate per IUI cycle of $12 \%$ for couples with that count. Wainer et al. reported on the number of motile sperm inseminated [50] and found a clinical pregnancy rate per cycle of $3.13 \%$ when the number of motile sperm inseminated was $<1 \times 10^{6}$. When the number of motile sperm inseminated was between 5 and $10 \times 10^{6}$, the clinical pregnancy rate per cycle was $14 \%$. Miller et al. reported a cutoff point for number of motile sperm inseminated after sperm preparation as $10 \times 10^{6}[51]$. Berg et al. reported successful pregnancies in patients after controlled ovarian stimulations in IUI preparations with motile sperm counts as low as $>0.8 \times 10^{6}[52]$.

Normal sperm morphology as a predictor of IUI success has been examined. After controlling for sperm concentration and motility, Lee et al. found that the normal sperm morphology, using Kruger's strict criteria [53], more strongly predicted IUI outcome [54]. They reported pregnancy rates per cycle of $3.8 \%$ in couples with $<4 \%$ of Kruger's strict criteria normal morphology, $18.5 \%$ for Kruger's strict criteria normal morphology of $4-9 \%$ and $29.9 \%$ when Kruger's strict criteria normal morphology was above $9 \%$ [54]. These results were similar to those reported in a study by Hauser et al. [55], where CC-IUI resulted in a pregnancy rates per couple of $11.1 \%$ when normal morphology by strict criteria was less than $4 \%, 36.1 \%$ for normal morphology of $4-14 \%$ and $50 \%$ when normal morphology was above $14 \%$. The link between sperm motility and IUI success was addressed in several studies. Briefly, in these studies, total motile sperm of $30-50 \%$ before sperm preparation was found to be associated with positive IUI outcomes $[54,56$, 57].

Considerations in Technology-Limited Settings. The evidence reviewed suggests that IUI may be offered to couples with male factor infertility, in a low technology setting, if the total motile sperm count is more than 5 million per specimen. 
TABLE 1: Motile sperm concentration, normal morphology, and IUI outcome.

\begin{tabular}{|c|c|c|c|c|c|c|c|c|}
\hline Author & Year & $\begin{array}{c}\text { Design } \\
\text { (prospective or } \\
\text { retrospective) }\end{array}$ & $\begin{array}{l}\text { Number of } \\
\text { infertile } \\
\text { patients }\end{array}$ & $\begin{array}{c}\text { Number of } \\
\text { insemination } \\
\text { cycles }\end{array}$ & $\begin{array}{c}\text { IMSC } \\
\text { (million) }\end{array}$ & $\begin{array}{c}\text { Normal } \\
\text { morphology }\end{array}$ & $\begin{array}{c}\text { IUI } \\
\text { outcome }\end{array}$ & $\begin{array}{c}\text { Total motile } \\
\text { sperm } \\
\text { count*10/6 } \\
\text { (before wash) }\end{array}$ \\
\hline \multirow[t]{3}{*}{ Ombelet et al. } & 1997 & Retrospective & 373 & 792 & $<1$ & $<4 \%$ & $0 \% \mathrm{CF}$ & \\
\hline & & & & & $<1$ & $>4 \%$ & $7.8 \%$ & \\
\hline & & & & & $>1$ & $>4 \%$ & $15 \%$ & \\
\hline \multirow[t]{2}{*}{ Van Voorhis et al. } & 2001 & Retrospective & 1,039 & 3,479 & & & PR $2.3 \%$ & $<10$ \\
\hline & & & & & & & PR 9\% & $>10$ \\
\hline \multirow[t]{2}{*}{ Cohlen et al. } & 1998 & Prospective & 74 & 308 & $<1$ & & PR $2.6 \%$ & \\
\hline & & & & & $>1$ & & PR $11.2 \%$ & \\
\hline \multirow[t]{2}{*}{ Wainer et al. } & 2004 & Retrospective & 889 & 2,564 & $<1$ & $>30 \%$ & PR $3.1 \%$ & \\
\hline & & & & & $2-5$ & & PR 12\% & \\
\hline \multirow[t]{2}{*}{ Dodson \& Haney } & 1991 & Retrospective & 371 & 808 & $<1$ & & CF $0 \%$ & \\
\hline & & & & & $1-5$ & & CF $17 \%$ & \\
\hline \multirow[t]{2}{*}{ Horvath et al. } & 1989 & Prospective & 232 & 451 & $<1$ & & CF $2.6 \%$ & \\
\hline & & & & & $>10$ & & CF $11.6 \%$ & \\
\hline \multirow[t]{2}{*}{ Berg et al. } & 1997 & Retrospective & 902 & 3,037 & $0.8-1.2$ & & PRcy $8.2 \%$ & \\
\hline & & & & & $>5$ & & $10 \%$ & \\
\hline \multirow[t]{3}{*}{ Miller et al. } & 2002 & Prospective & 438 & 1,114 & $<10$ & & CF $1 \%$ & \\
\hline & & & & & $10-20$ & & CF $7.4 \%$ & \\
\hline & & & & & $>20$ & & CF $12.4 \%$ & \\
\hline Irianni et al. & 1993 & Retrospective & 129 & 208 & $>4.7$ & & PR 14\% & \\
\hline Toner et al. & 1994 & Prospective & 126 & 395 & 104 & $9 \%$ & PRcy 9\% & \\
\hline \multirow[t]{2}{*}{ Lindheim et al. } & 1996 & Retrospective & 42 & & & $<4 \%$ & PRcy 1\% & \\
\hline & & & & & & $>4 \%$ & PRcy 19\% & \\
\hline \multirow[t]{3}{*}{ Montanaro Gauci et al. } & 1999 & Retrospective & 273 & 522 & & $<4 \%$ & PRcy $2.6 \%$ & \\
\hline & & & & & & $5-14 \%$ & $11 \%$ & \\
\hline & & & & & & $>14 \%$ & $24 \%$ & \\
\hline Shibahara et al. & 2004 & Prospective & 160 & 682 & & $>15 \%$ & PRcy $15 \%$ & \\
\hline \multirow[t]{3}{*}{ Hauser et al. } & 2001 & Prospective & 108 & 264 & & $<4 \%$ & $\begin{array}{c}\text { 4-PRcp } \\
11 \%\end{array}$ & \\
\hline & & & & & & $14 \%$ & $36 \%$ & \\
\hline & & & & & & $>14 \%$ & $50 \%$ & \\
\hline \multirow[t]{3}{*}{ Lee et al. } & 2002 & Prospective & 209 & 244 & & $<4 \%$ & PRcy 3.8\% & \\
\hline & & & & & & $4-9 \%$ & $18.5 \%$ & \\
\hline & & & & & & $>9 \%$ & $30 \%$ & \\
\hline \multirow[t]{2}{*}{ Dickey et al. } & 1999 & Retrospective & 1,841 & 4,056 & & $<5 \%$ & PRcy $0 \%$ & \\
\hline & & & & & & $>5 \%$ & $11 \%$ & \\
\hline \multirow[t]{2}{*}{ Dickey et al. } & 1999 & Retrospective & 1,841 & 4,056 & & & PRcy $2.3 \%$ & $<5$ \\
\hline & & & & & & & $8.5 \%$ & $>5$ \\
\hline
\end{tabular}




\section{Sperm Preparation Methods}

The aim of semen preparation is to separate motile, morphologically normal spermatozoa from the seminal plasma and from debris such as leucocytes, bacteria, and nonmotile spermatozoa. Table 2 shows studies that addressed the effect of different sperm preparation methods on IUI outcome. As shown in the table, the number of these studies is limited. Sperm wash, swim-up, and density gradient centrifugation are the most commonly used methods. As shown in Table 2, density gradient centrifugation was associated with acceptable IUI outcomes [58-62]. Swim-up as a procedure is also associated with satisfactory IUI outcomes, comparable to density gradient centrifugation pregnancy rates. Furthermore, other studies (the preparation technique was not the primary question addressed) reported similar outcomes for both methods $[47,50]$. Boomsma et al. conducted a Cochrane review to compare the effectiveness of the three different semen preparation techniques (conventional wash, swim-up procedure, and density gradient centrifugation) before IUI [63]. Only two RCTs could be included in the meta-analysis regarding the clinical outcomes. This resulted in insufficient data to recommend anyone of the three semen preparation techniques over the others.

The time intervals between semen collection, sperm processing, and insemination may affect IUI outcomes. Yavas and Selub performed a retrospective study where the collection of semen at the clinic was found to be better than at home. Short intervals from semen collection to sperm wash, from sperm wash to IUI, and from semen collection to IUI were associated with higher pregnancy rates compared to semen collection at home with longer time intervals [64].

Considerations in Technology-Limited Settings. The evidence suggests that conventional sperm wash for IUI can be used in a low-technology setting. The evidence does not seem to show that anyone of the three commonly used sperm preparation methods results in a higher pregnancy rate than any other.

\section{Timing of IUI}

Normal sperm is capable of fertilizing an oocyte in the female genital tract for about 5 days, and an oocyte is fertilizable for 12-24 hours after ovulation [65]. The WHO conducted a large multicenter study that concluded that ovulation occurred between 24 and 56 hours (average 32 hours) after the onset of luteinizig hormone (LH) surge [66]. In another study ovulation, occurred 36-38 hours after the onset of LH surge in natural cycles [67]. The LH surge can be detected at home using urinary LH kits. The monitoring for the urinary LH surge can start about 3 days before the expected date of ovulation. Generally, in natural cycles, IUI can be performed 24 hours after the onset of LH surge as detected by urinary LH monitoring.

In controlled ovarian stimulation cycles in which ovulation is triggered artificially, ovulation occurs $32-38$ hours after human chorionic gonadotropin (HCG) injection [68,
69]. Based on the available data about the timing of ovulation, some investigators recommended that insemination can be carried out between 12 and 60 hours after HCG injection [70-72]. Other investigators found that delayed inseminations to 38-40 hours after HCG injection were associated with higher pregnancy rates compared to early inseminations [73]. This was not found in a study by Claman et al., who found that there was no significant difference in pregnancy rates between early (32-34 hours) and late (38-40 hours) inseminations [74]. When HCG is used, insemination can be done 34-40 hours after injection [75].

A systematic review was performed by Kosmas et al. to compare the effectiveness of HCG administration versus urinary LH detection as a method of IUI timing after CC stimulation [76]. Seven studies were included in this systematic review and the meta-analysis. The authors found that the use of urinary LH monitoring as a method of IUI timing was associated with higher pregnancy rates than the HCG administration method. They concluded that LH monitoring for IUI timing is more practical, effective, and cost-effective when CC is used for ovarian stimulation [76].

Considerations in Technology-Limited Settings. The evidence suggests that it is reasonable to perform IUI, for either natural cycle IUI or for clomiphene combined with IUI, approximately 24 hours after the detection of the LH surge. Urine testing kits could be used to detect the LH surge.

\section{Number of Inseminations per Cycle}

Several studies have compared single versus double IUIs in an ovulatory cycle. One of the earlier studies, conducted for that purpose, reported a cycle fecundity of $52 \%$ when 2 inseminations 18 and 42 hours after HCG were performed while a single insemination performed 34 hours after HCG injection resulted in a cycle fecundity of $8.7 \%$ [71]. Another study found that double inseminations, performed 12 and 34 hours after HCG administration, in gonadotropin stimulated cycles, resulted in a pregnancy rate per patient of $30.4 \%$, a higher pregnancy rate than that found from single inseminations (14.4\%) performed 34 hours after HCG [72].

On the other hand, Ransom et al. found no difference in pregnancy rates between single IUI performed 35 hours after HCG and double IUI done 19 and 43 hours after HCG injection [77]. These results were supported in a study by Alborzi et al. [78] where pregnancy rates per cycle were 8.6\% and 9.4\% (nonsignificantly different) for single and double IUIs. Another study, by Casadei et al., failed to show a significant advantage of double inseminations 12 and 36 hours after HCG injection over single insemination done 36 hours after HCG [79].

Two reviews were conducted to reach a conclusion about the number of inseminations per cycle [80]. One was a systematic review and meta-analysis [80] and the second was a Cochrane analysis [81]. In the first review, by Osuna et al., 18 trials were retrieved from searching Medline, The Cochrane library, and the abstract books of annual meetings of ESHRE and ASRM [80]. Only six randomized prospective 
TABLE 2: Sperm preparation methods.

\begin{tabular}{|c|c|c|c|c|c|c|}
\hline Author & Year & $\begin{array}{l}\text { Design (prospective } \\
\text { or retrospective) }\end{array}$ & $\begin{array}{l}\text { Number of } \\
\text { infertile patients }\end{array}$ & $\begin{array}{l}\text { Number of } \\
\text { insemination cycles }\end{array}$ & $\begin{array}{l}\text { Method of } \\
\text { preparation }\end{array}$ & IUI outcome \\
\hline \multirow{5}{*}{ Carrell et al. } & \multirow{5}{*}{1998} & \multirow{5}{*}{ Prospective } & \multirow{5}{*}{363} & \multirow{5}{*}{898} & Sperm wash & PR $8.9 \%$ \\
\hline & & & & & -Swim-up & $14.7 \%$ \\
\hline & & & & & -Swim-down & $7.7 \%$ \\
\hline & & & & & $\begin{array}{l}\text {-Percoll density } \\
\text { gradient }\end{array}$ & $16.1 \%$ \\
\hline & & & & & $\begin{array}{l}\text {-Sperm refriger- } \\
\text { ation/heparin } \\
\text { incubation }\end{array}$ & $11 \%$ \\
\hline \multirow{3}{*}{ Dodson et al. } & \multirow{3}{*}{1998} & \multirow{3}{*}{ Prospective } & \multirow{3}{*}{80} & \multirow{3}{*}{153} & $\begin{array}{l}\text { Double } \\
\text { cenrifugation }\end{array}$ & CF $15 \%$ \\
\hline & & & & & -Swim-up & $14 \%$ \\
\hline & & & & & $\begin{array}{l}\text {-Percoll density } \\
\text { gradient }\end{array}$ & $20 \%$ \\
\hline \multirow[t]{2}{*}{ Morshedi et al. } & \multirow{2}{*}{2003} & \multirow{2}{*}{ Prospective } & \multirow{2}{*}{311} & \multirow{2}{*}{676} & Simple wash & PR $11.6 \%$ \\
\hline & & & & & $\begin{array}{l}\text {-Density } \\
\text { gradient }\end{array}$ & $14.3 \%$ \\
\hline \multirow{3}{*}{ Lan et al. } & \multirow{3}{*}{2004} & \multirow{3}{*}{ Prospective } & \multirow{3}{*}{140} & \multirow{3}{*}{ NA } & Swim-up & PR $15 \%$ \\
\hline & & & & & $\begin{array}{l}\text {-Percoll density } \\
\text { gradient }\end{array}$ & $20 \%$ \\
\hline & & & & & $\begin{array}{l}\text {-Wang's tube } \\
\text { method }\end{array}$ & $45 \%$ \\
\hline \multirow{4}{*}{ Ren et al. } & \multirow{4}{*}{2004} & \multirow{4}{*}{ Prospective } & \multirow{4}{*}{ NA } & \multirow{4}{*}{317} & Albumin & PR $12.5 \%$ \\
\hline & & & & & -Puresperm & $7.4 \%$ \\
\hline & & & & & -Swim-up & $9.6 \%$ \\
\hline & & & & & $\begin{array}{l}\text {-Percoll density } \\
\text { gradient }\end{array}$ & $12.7 \%$ \\
\hline
\end{tabular}

PR: Pregnancy rate; CF: Cycle fecundity; NA: Not available.

studies were included in the review. The pooled outcomes of the studies were a pregnancy rate per cycle of $14.9 \%$ for double IUIs versus $11.4 \%$ for a single IUI. This difference was statistically insignificant. The Cochrane review, by Cantineau et al., followed the same search strategies and data analyses as mentioned previously [81]. From the 30 studies retrieved, 13 were found to provide data comparing single versus double inseminations. Only 3 RCTs were included in the review, the results of 2 of them were pooled for metaanalysis. The authors concluded that there was no evidence that double inseminations give rise to higher live birth rates in infertile couples compared to single inseminations (OR 1.45, CI: 0.78-2.7). The NICE recommendation is for a single insemination when offering IUI as therapy [39].

Considerations in Technology-Limited Settings. The evidence suggests that single IUI insemination per ovulatory cycle should be considered. Published evidence does not support that two IUI in a single cycle results in a higher rate of pregnancy. A single insemination will be less costly, with similar pregnancy outcomes.

\section{Number of Insemination Cycles}

Some studies report six treatment cycles $[29,60,78]$, while others report four cycles $[35,82]$ as the number of cycles of treatment with IUIs. One study reported a cumulative probability of pregnancy of $38.2 \%$ for 6 treatment cycles and $49.5 \%$ for 10 treatment cycles [52]. One of the sixcycle studies, by Morshedi et al., reported that $88 \%$ of the pregnancies occurred in the first 3 cycles [60]. However, a Cochrane review and the NICE guidelines support using IUI as treatment for up to 6 cycles $[32,83]$. The NICE reported, with level I evidence, that the use of up to 6 cycles on IUI increased the chance of pregnancy for couples with mild male-factor and unexplained infertility and minimal to mild endometriosis. The Cochrane reviewers concluded that most pregnancies occurred during the first 3 to 6 IUIs treatment cycles.

Considerations in Technology-Limited Settings. The evidence suggests performing a minimum of three IUI treatment cycles and a maximum of six IUI treatment cycles in technology-limited setting. 


\section{Conclusion}

The resource allocation of infertility services available for patients is asymmetric, with the highest levels in the larger cities. The evidence reviewed suggests that IUI may be helpful in a low-technology medical setting. It may be considered as a good first-line treatment for couples with unexplained infertility, male factor infertility, and anovulation (IUI used concurrently with ovulation induction). In the clinical practice of IUI in a low-technology setting, combining oral clomiphene with IUI is as reasonable of an option as natural cycle IUI. For semen parameters, a motile sperm count above 1 million in the final specimen can serve as a cutoff point for offering IUI. Conventional sperm washing, density gradient centrifugation, or swimup techniques can all be used for sperm preparation before IUI, with conventional sperm washing being the simplest. A single IUI per cycle should be performed, and the IUI can be performed approximately 24 hours after urinary LH surge is detected. Couples may be offered 3 to 6 IUI cycles to ensure sufficient opportunity to achieve pregnancy.

\section{References}

[1] C. Keck, C. Gerber-Schafer, C. Wilhelm, D. Vogelgesang, and M. Breckwoldt, "Intrauterine insemination for treatment of male infertility," International Journal of Andrology, vol. 20, supplement 3, pp. 55-64, 1997.

[2] W. Ombelet, K. Deblaere, E. Bosmans, et al., "Semen quality and intrauterine insemination," Reproductive BioMedicine Online, vol. 7, no. 4, pp. 485-492, 2003.

[3] J. U. Klein, D. Kuleta, and J. C. Petrozza, "Intrauterine insemination (IUI) outcomes in cases performed by nurses as compared with cases performed by physicians," Fertility and Sterility, vol. 88, supplement 1, pp. S9-S10, 2007.

[4] J. Boivin, L. Bunting, J. A. Collins, and K. G. Nygren, "International estimates of infertility prevalence and treatmentseeking: potential need and demand for infertility medical care," Human Reproduction, vol. 22, no. 6, pp. 1506-1512, 2007.

[5] E. H. Stephen and A. Chandra, "Use of infertility services in the United States: 1995," Family Planning Perspectives, vol. 32, no. 3, pp. 132-137, 2000.

[6] J. C. Abma, A. Chandra, W. D. Mosher, L. S. Peterson, and L. J. Piccinino, "Fertility, family planning, and women's health: new data from the 1995 National Survey of Family Growth," Vital and Health Statistics, vol. 23, no. 19, pp. 1-114, 1997.

[7] D. W. Stovall, B. D. Allen, A. E. T. Sparks, C. H. Syrop, R. G. Saunders, and B. J. Van Voorhis, "The cost of infertility evaluation and therapy: findings of a self-insured university healthcare plan," Fertility and Sterility, vol. 72, no. 5, pp. 778784, 1999.

[8] B. J. Oddens, I. den Tonkelaar, and H. Nieuwenhuyse, "Psychosocial experiences in women facing fertility problems-a comparative survey," Human Reproduction, vol. 14, no. 1, pp. 255-261, 1999.

[9] A. D. Domar, P. C. Zuttermeister, and R. Friedman, "The psychological impact of infertility: a comparison with patients with other medical conditions," Journal of Psychosomatic Obstetrics and Gynaecology, vol. 14, supplement, pp. 45-52, 1993.
[10] P. Thiering, J. Beaurepaire, M. Jones, D. Saunders, and C. Tennant, "Mood state as a predictor of treatment outcome after in vitro fertilization/embryo transfer technology (IVF/ET)," Journal of Psychosomatic Research, vol. 37, no. 5, pp. 481-491, 1993.

[11] Society for Assisted Reproductive Technology, American Society for Reproductive Medicine, "Assisted reproductive technology in the United States: 2001 results generated from the American Society for Reproductive Medicine/Society for Assisted Reproductive Technology registry," Fertility and Sterility, vol. 87, no. 6, pp. 1253-1266, 2007.

[12] A. Nyboe Andersen, V. Goossens, L. Gianaroli, R. Felberbaum, J. de Mouzon, and K. G. Nygren, "Assisted reproductive technology in Europe, 2003. Results generated from European registers by ESHRE," Human Reproduction, vol. 22, no. 6, pp. 1513-1525, 2007.

[13] J. Collins, "Cost-effectiveness of in vitro fertilization," Seminars in Reproductive Medicine, vol. 19, no. 3, pp. 279-289, 2001.

[14] U. M. Reddy, R. J. Wapner, R. W. Rebar, and R. J. Tasca, "Infertility, assisted reproductive technology, and adverse pregnancy outcomes: executive summary of a National Institute of Child Health and Human Development workshop," Obstetrics and Gynecology, vol. 109, no. 4, pp. 967-977, 2007.

[15] V. C. Wright, J. Chang, G. Jeng, and M. Macaluso, "Assisted reproductive technology surveillance-United States, 2003," MMWR. Surveillance Summaries, vol. 55, no. SS04, pp. 1-22, 2006.

[16] R. A. Jackson, K. A. Gibson, Y. W. Wu, and M. S. Croughan, "Perinatal outcomes in singletons following in vitro fertilization: a meta-analysis," Obstetrics and Gynecology, vol. 103, no. 3, pp. 551-563, 2004.

[17] A. Schreurs, E. Legius, C. Meuleman, J.-P. Fryns, and T. M. D'Hooghe, "Increased frequency of chromosomal abnormalities in female partners of couples undergoing in vitro fertilization or intracytoplasmic sperm injection," Fertility and Sterility, vol. 74, no. 1, pp. 94-96, 2000.

[18] M. Hansen, J. J. Kurinczuk, C. Bower, and S. Webb, "The risk of major birth defects after intracytoplasmic sperm injection and in vitro fertilization," The New England Journal of Medicine, vol. 346, no. 10, pp. 725-730, 2002.

[19] W. Ombelet, P. Puttemans, and E. Bosmans, "Intrauterine insemination: a first-step procedure in the algorithm of male subfertility treatment," Human Reproduction, vol. 10, supplement 1, pp. 90-102, 1995.

[20] M. Gaudoin, R. Dobbie, A. Finlayson, J. Chalmers, I. T. Cameron, and R. Fleming, "Ovulation induction/intrauterine insemination in infertile couples is associated with low-birthweight infants," American Journal of Obstetrics \& Gynecology, vol. 188 , no. 3, pp. 611-616, 2003.

[21] S. Nuojua-Huttunen, M. Gissler, H. Martikainen, and L. Tuomivaara, "Obstetric and perinatal outcome of pregnancies after intrauterine insemination," Human Reproduction, vol. 14, no. 8, pp. 2110-2115, 1999.

[22] J. Hoy, A. Venn, J. Halliday, G. Kovacs, and K. Waalwyk, "Perinatal and obstetric outcomes of donor insemination using cryopreserved semen in Victoria, Australia," Human Reproduction, vol. 14, no. 7, pp. 1760-1764, 1999.

[23] N. C. Allen, C. M. Herbert III, W. S. Maxson, B. J. Rogers, M. P. Diamond, and A. C. Wentz, "Intrauterine insemination: a critical review," Fertility and Sterility, vol. 44, no. 5, pp. 569$580,1985$.

[24] K. Ahinko-Hakamaa, H. Huhtala, and H. Tinkanen, "Success in intrauterine insemination: the role of etiology," Acta 
Obstetricia et Gynecologica Scandinavica, vol. 86, no. 7, pp. 855-860, 2007.

[25] M. R. Khalil, P. E. Rasmussen, K. Erb, S. B. Laursen, S. Rex, and L. G. Westergaard, "Homologous intrauterine insemination. An evaluation of prognostic factors based on a review of 2473 cycles," Acta Obstetricia et Gynecologica Scandinavica, vol. 80, no. 1, pp. 74-81, 2001.

[26] M. J. Tomlinson, J. B. Amissah-Arthur, K. A. Thompson, J. L. Kasraie, and B. Bentick, "Prognostic indicators for intrauterine insemination (IUI): statistical model for IUI success," Human Reproduction, vol. 11, no. 9, pp. 1892-1896, 1996.

[27] B. J. Van Voorhis, A. E. T. Sparks, B. D. Allen, D. W. Stovall, C. H. Syrop, and F. K. Chapler, "Cost-effectiveness of infertility treatments: a cohort study," Fertility and Sterility, vol. 67, no. 5, pp. 830-836, 1997.

[28] V. C. Karande, A. Korn, R. Morris, et al., "Prospective randomized trial comparing the outcome and cost of in vitro fertilization with that of a traditional treatment algorithm as first-line therapy for couples with infertility," Fertility and Sterility, vol. 71, no. 3, pp. 468-475, 1999.

[29] A. J. Goverde, J. McDonnell, J. P. W. Vermeiden, R. Schats, F. F. H. Rutten, and J. Schoemaker, "Intrauterine insemination or in-vitro fertilisation in idiopathic subfertility and male subfertility: a randomised trial and cost-effectiveness analysis," The Lancet, vol. 355, no. 9197, pp. 13-18, 2000.

[30] C. M. Peterson, H. H. Hatasaka, K. P. Jones, A. M. Poulson Jr., D. T. Carrell, and R. L. Urry, "Ovulation induction with gonadotropins and intrauterine insemination compared with in vitro fertilization and no therapy: a prospective, nonrandomized, cohort study and meta-analysis," Fertility and Sterility, vol. 62, no. 3, pp. 535-544, 1994.

[31] F. Zayed, E. A. Lenton, and I. D. Cooke, "Comparison between stimulated in-vitro fertilization and stimulated intrauterine insemination for the treatment of unexplained and mild male factor infertility," Human Reproduction, vol. 12, no. 11, pp. 2408-2413, 1997.

[32] B. J. Cohlen, P. Vandekerckhove, E. R. te Velde, and J. D. Habbema, "Timed intercourse versus intra-uterine insemination with or without ovarian hyperstimulation for subfertility in men," The Cochrane Database of Systematic Reviews, no. 4, Article ID CD000360, 2000.

[33] N. Pashayan, G. Lyratzopoulos, and R. Mathur, "Costeffectiveness of primary offer of IVF vs. primary offer of IUI followed by IVF (for IUI failures) in couples with unexplained or mild male factor subfertility," BMC Health Services Research, vol. 6, article 80, pp. 1-11, 2006.

[34] E. G. Hughes, "The effectiveness of ovulation induction and intrauterine insemination in the treatment of persistent infertility: a meta-analysis," Human Reproduction, vol. 12, no. 9, pp. 1865-1872, 1997.

[35] D. S. Guzick, S. A. Carson, C. Coutifaris, et al., "Efficacy of superovulation and intrauterine insemination in the treatment of infertility," The New England Journal of Medicine, vol. 340, no. 3, pp. 177-183, 1999.

[36] S. M. Verhulst, B. J. Cohlen, E. Hughes, M. J. Heineman, and E. te Velde, "Intra-uterine insemination for unexplained subfertility," The Cochrane Database of Systematic Reviews, no. 4, Article ID CD001838, pp. 1-82, 2006.

[37] P. Steures, J. W. van der Steeg, P. G. Hompes, et al., "Intrauterine insemination with controlled ovarian hyperstimulation versus expectant management for couples with unexplained subfertility and an intermediate prognosis: a randomised clinical trial," The Lancet, vol. 368, no. 9531, pp. 216-221, 2006.

[38] P. Steures, J. W. van der Steeg, P. G. A. Hompes, et al., "The additional value of ovarian hyperstimulation in intrauterine insemination for couples with an abnormal postcoital test and a poor prognosis: a randomized clinical trial," Fertility and Sterility, vol. 88, no. 6, pp. 1618-1624, 2007.

[39] NICE Guidelines: National Institute for Clinical Excellence, Fertility: Assessment and Treatment for People with Fertility Problems, RCOG Press, London, UK, 2004.

[40] G. Ragni, F. Alagna, C. Brigante, et al., "GnRH antagonists and mild ovarian stimulation for intrauterine insemination: a randomized study comparing different gonadotrophin dosages," Human Reproduction, vol. 19, no. 1, pp. 54-58, 2004.

[41] S. Gerli, M. L. Casini, V. Unfer, L. Costabile, V. Bini, and G. C. Di Renzo, "Recombinant versus urinary follicle-stimulating hormone in intrauterine insemination cycles: a prospective, randomized analysis of cost effectiveness," Fertility and Sterility, vol. 82, no. 3, pp. 573-578, 2004.

[42] A. Demirol and T. Gurgan, "Comparison of different gonadotrophin preparations in intrauterine insemination cycles for the treatment of unexplained infertility: a prospective, randomized study," Human Reproduction, vol. 22, no. 1, pp. 97-100, 2007.

[43] T. Dankert, J. A. Kremer, B. J. Cohlen, et al., "A randomized clinical trial of clomiphene citrate versus low dose recombinant FSH for ovarian hyperstimulation in intrauterine insemination cycles for unexplained and male subfertility," Human Reproduction, vol. 22, no. 3, pp. 792-797, 2007.

[44] A. E. P. Cantineau and B. J. Cohlen, "Ovarian stimulation protocols (anti-oestrogens, gonadotrophins with and without $\mathrm{GnRH}$ agonists/antagonists) for intrauterine insemination (IUI) in women with subfertility," The Cochrane Database of Systematic Reviews, no. 2, Article ID CD005356, pp. 1-135, 2007.

[45] M. F. Costello, "Systematic review of the treatment of ovulatory infertility with clomiphene citrate and intrauterine insemination," Australian and New Zealand Journal of Obstetrics and Gynaecology, vol. 44, no. 2, pp. 93-102, 2004.

[46] World Health Organization, "Collection and examination of human semen," in WHO Laboratory Manual for the Examination of Human Semen and Sperm-Cervical Mucus Interaction, pp. 4-33, Cambridge University Press, Cambridge, UK, 4th edition, 1999.

[47] W. Ombelet, H. Vandeput, G. Van de Putte, et al., "Intrauterine insemination after ovarian stimulation with clomiphene citrate: predictive potential of inseminating motile count and sperm morphology," Human Reproduction, vol. 12, no. 7, pp. 1458-1463, 1997.

[48] B. J. Van Voorhis, M. Barnett, A. E. T. Sparks, C. H. Syrop, G. Rosenthal, and J. Dawson, "Effect of the total motile sperm count on the efficacy and cost-effectiveness of intrauterine insemination and in vitro fertilization," Fertility and Sterility, vol. 75, no. 4, pp. 661-668, 2001.

[49] B. J. Cohlen, E. R. te Velde, R. J. van Kooij, C. W. N. Looman, and J. D. F. Habbema, "Controlled ovarian hyperstimulation and intrauterine insemination for treating male subfertility: a controlled study," Human Reproduction, vol. 13, no. 6, pp. 1553-1558, 1998.

[50] R. Wainer, M. Albert, A. Dorion, et al., "Influence of the number of motile spermatozoa inseminated and of their morphology on the success of intrauterine insemination," Human Reproduction, vol. 19, no. 9, pp. 2060-2065, 2004. 
[51] D. C. Miller, B. K. Hollenbeck, G. D. Smith, et al., "Processed total motile sperm count correlates with pregnancy outcome after intrauterine insemination," Urology, vol. 60, no. 3, pp. 497-501, 2002.

[52] U. Berg, C. Brucker, and F. D. Berg, "Effect of motile sperm count after swim-up on outcome of intrauterine insemination," Fertility and Sterility, vol. 67, no. 4, pp. 747750, 1997.

[53] T. F. Kruger, A. A. Acosta, K. F. Simmons, R. J. Swanson, J. F. Matta, and S. Oehninger, "Predictive value of abnormal sperm morphology in in vitro fertilization," Fertility and Sterility, vol. 49, no. 1, pp. 112-117, 1988.

[54] R. K.-K. Lee, J.-W. Hou, H.-Y. Ho, et al., "Sperm morphology analysis using strict criteria as a prognostic factor in intrauterine insemination," International Journal of Andrology, vol. 25, no. 5, pp. 277-280, 2002.

[55] R. Hauser, L. Yogev, A. Botchan, J. B. Lessing, G. Paz, and H. Yavetz, "Intrauterine insemination in male factor subfertility: significance of sperm motility and morphology assessed by strict criteria," Andrologia, vol. 33, no. 1, pp. 13-17, 2001.

[56] M. Montanaro Gauci, T. F. Kruger, K. Coetzee, K. Smith, J. P. van der Merwe, and C. J. Lombard, "Stepwise regression analysis to study male and female factors impacting on pregnancy rate in an intrauterine insemination programme," Andrologia, vol. 33, no. 3, pp. 135-141, 2001.

[57] R. P. Dickey, R. Pyrzak, P. Y. Lu, S. N. Taylor, and P. H. Rye, "Comparison of the sperm quality necessary for successful intrauterine insemination with World Health Organization threshold values for normal sperm," Fertility and Sterility, vol. 71, no. 4, pp. 684-689, 1999.

[58] D. T. Carrell, P. H. Kuneck, C. M. Peterson, H. H. Hatasaka, K. P. Jones, and B. F. Campbell, "A randomized, prospective analysis of five sperm preparation techniques before intrauterine insemination of husband sperm," Fertility and Sterility, vol. 69 , no. 1 , pp. 122-126, 1998.

[59] W. C. Dodson, J. Moessner, J. Miller, R. S. Legro, and C. L. Gnatuk, "A randomized comparison of the methods of sperm preparation for intrauterine insemination," Fertility and Sterility, vol. 70, no. 3, pp. 574-575, 1998.

[60] M. Morshedi, H. E. Duran, S. Taylor, and S. Oehninger, "Efficacy and pregnancy outcome of two methods of semen preparation for intrauterine insemination: a prospective randomized study," Fertility and Sterility, vol. 79, supplement 3, pp. 1625-1632, 2003.

[61] L. Xu, R.-K. Lu, L. Chen, and Y.-L. Zheng, "Comparative study on efficacy of three sperm-separation techniques," Asian Journal of Andrology, vol. 2, no. 2, pp. 131-134, 2000.

[62] S.-S. Ren, G.-H. Sun, C.-H. Ku, D.-C. Chen, and G.-J. Wu, "Comparison of four methods for sperm preparation for IUI," Archives of Andrology, vol. 50, no. 3, pp. 139-143, 2004.

[63] C. M. Boomsma, M. J. Heineman, B. J. Cohlen, and C. Farquhar, "Semen preparation techniques for intrauterine insemination," The Cochrane Database of Systematic Reviews, no. 4, Article ID CD004507, pp. 1-29, 2007.

[64] Y. Yavas and M. R. Selub, "Intrauterine insemination (IUI) pregnancy outcome is enhanced by shorter intervals from semen collection to sperm wash, from sperm wash to IUI time, and from semen collection to IUI time," Fertility and Sterility, vol. 82, no. 6, pp. 1638-1647, 2004.

[65] J. P. Royston, "Basal body temperature, ovulation and the risk of conception, with special reference to the lifetimes of sperm and egg," Biometrics, vol. 38, no. 2, pp. 397-406, 1982.

[66] World Health Organization, "Temporal relationships between ovulation and defined changes in the concentration of plasma estradiol-17 $\beta$, luteinizing hormone, follicle, stimulating hormone and progesterone," American Journal of Obstetrics \& Gynecology, vol. 138, no. 4, pp. 383-390, 1980.

[67] M. L. Taymor, M. M. Seibel, D. Smith, and L. Levesque, "Ovulation timing by luteinizing hormone assay and follicle puncture," Obstetrics and Gynecology, vol. 62, no. 2, pp. 191195, 1983.

[68] R. G. Edwards and P. C. Steptoe, "Control of human ovulation, fertilization and implantation," Proceedings of the Royal Society of Medicine, vol. 67, no. 9, pp. 932-936, 1974.

[69] J. Testart and R. Frydman, "Minimum time lapse between luteinizing hormone surge or human chorionic gonadotropin administration and follicular rupture," Fertility and Sterility, vol. 37, no. 1, pp. 50-53, 1982.

[70] K. W. Fuh, X. Wang, A. Tai, I. Wong, and R. J. Norman, "Intrauterine insemination: effect of the temporal relationship between the luteinizing hormone surge, human chorionic gonadotrophin administration and insemination on pregnancy rates," Human Reproduction, vol. 12, no. 10, pp. 21622166, 1997.

[71] K. M. Silverberg, J. V. Johnson, D. L. Olive, W. N. Burns, and R. S. Schenken, "A prospective, randomized trial comparing two different intrauterine insemination regimens in controlled ovarian hyperstimulation cycles," Fertility and Sterility, vol. 57, no. 2, pp. 357-361, 1992.

[72] G. Ragni, P. Maggioni, E. Guermandi, et al., "Efficacy of double intrauterine insemination in controlled ovarian hyperstimulation cycles," Fertility and Sterility, vol. 72, no. 4, pp. 619-622, 1999.

[73] J. L. Pryor, P. H. Kuneck, S. M. Blatz, C. Thorp, C. E. Cornwell, and D. T. Carrell, "Delayed timing of intrauterine insemination results in a significantly improved pregnancy rate in female partners of quadriplegic men," Fertility and Sterility, vol. 76, no. 6, pp. 1130-1135, 2001.

[74] P. Claman, V. Wilkie, and D. Collins, "Timing intrauterine insemination either 33 or 39 hours after administration of human chorionic gonadotropin yields the same pregnancy rates as after superovulation therapy," Fertility and Sterility, vol. 82, no. 1, pp. 13-16, 2004.

[75] L. Speroff and M. A. Fritz, "Male infertility," in Clinical Gynecologic Endocrinology and Infertility, L. Speroff and M. A. Fritz, Eds., pp. 1135-1173, Lippincott Williams and Wilkins, Philadelphia, Pa, USA, 2005.

[76] I. P. Kosmas, A. Tatsioni, H. M. Fatemi, E. M. Kolibianakis, H. Tournaye, and P. Devroey, "Human chorionic gonadotropin administration vs. luteinizing monitoring for intrauterine insemination timing, after administration of clomiphene citrate: a meta-analysis," Fertility and Sterility, vol. 87 , no. 3, pp. 607-612, 2007.

[77] M. X. Ransom, M. B. Blotner, M. Bohrer, G. Corsan, and E. Kemmann, "Does increasing frequency of intrauterine insemination improve pregnancy rates significantly during superovulation cycles?" Fertility and Sterility, vol. 61, no. 2, pp. 303-307, 1994.

[78] S. Alborzi, S. Motazedian, M. E. Parsanezhad, and S. Jannati, "Comparison of the effectiveness of single intrauterine insemination (IUI) versus double IUI per cycle in infertile patients," Fertility and Sterility, vol. 80, no. 3, pp. 595-599, 2003.

[79] L. Casadei, V. Zamaro, M. Calcagni, C. Ticconi, M. Dorrucci, and E. Piccione, "Homologous intrauterine insemination in controlled ovarian hyperstimulation cycles: a comparison among three different regimens," European Journal of Obstetrics Gynecology and Reproductive Biology, vol. 129, no. 2, pp. 155-161, 2006. 
[80] C. Osuna, R. Matorras, J. I. Pijoan, and F. J. RodríguezEscudero, "One versus two inseminations per cycle in intrauterine insemination with sperm from patients' husbands: a systematic review of the literature," Fertility and Sterility, vol. 82, no. 1, pp. 17-24, 2004.

[81] A. E. Cantineau, M. J. Heineman, and B. J. Cohlen, "Single versus double intrauterine insemination (IUI) in stimulated cycles for subfertile couples," The Cochrane Database of Systematic Reviews, no. 1, Article ID CD003854, pp. 1-32, 2003.

[82] P. Steures, J. W. van der Steeg, H. R. Verhoeve, et al., "Does ovarian hyperstimulation in intrauterine insemination for cervical factor subfertility improve pregnancy rates?" Human Reproduction, vol. 19, no. 10, pp. 2263-2266, 2004.

[83] NICE Guidelines, "Intrauterine insemination," in National Institute for Clinical Excellence Fertility: Assessment and Treatment for People with Fertility Problems, pp. 75-80, RCOG Press, London, UK, 2004. 


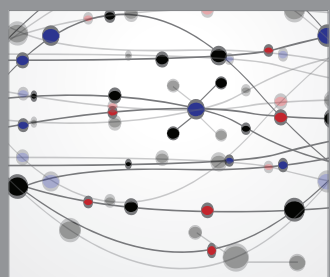

The Scientific World Journal
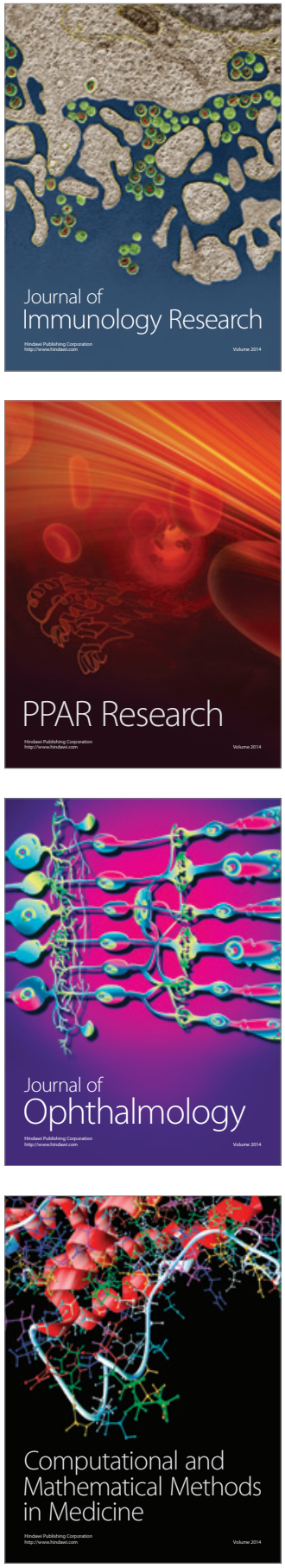

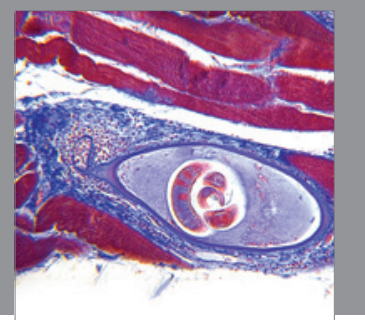

Gastroenterology

Research and Practice
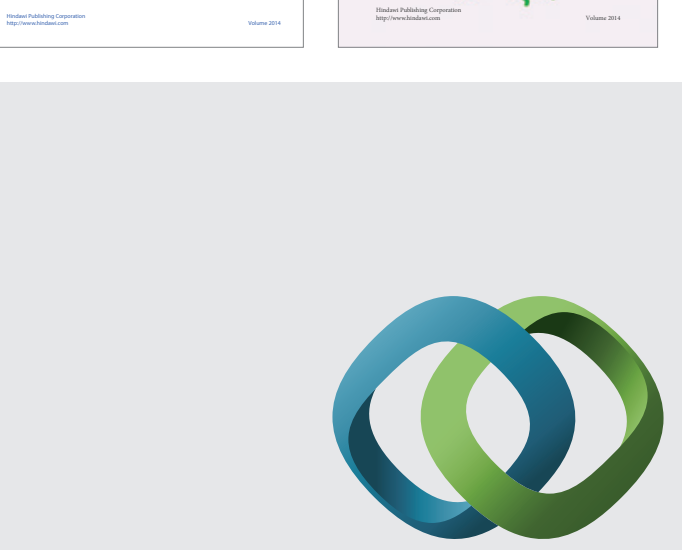

\section{Hindawi}

Submit your manuscripts at

http://www.hindawi.com
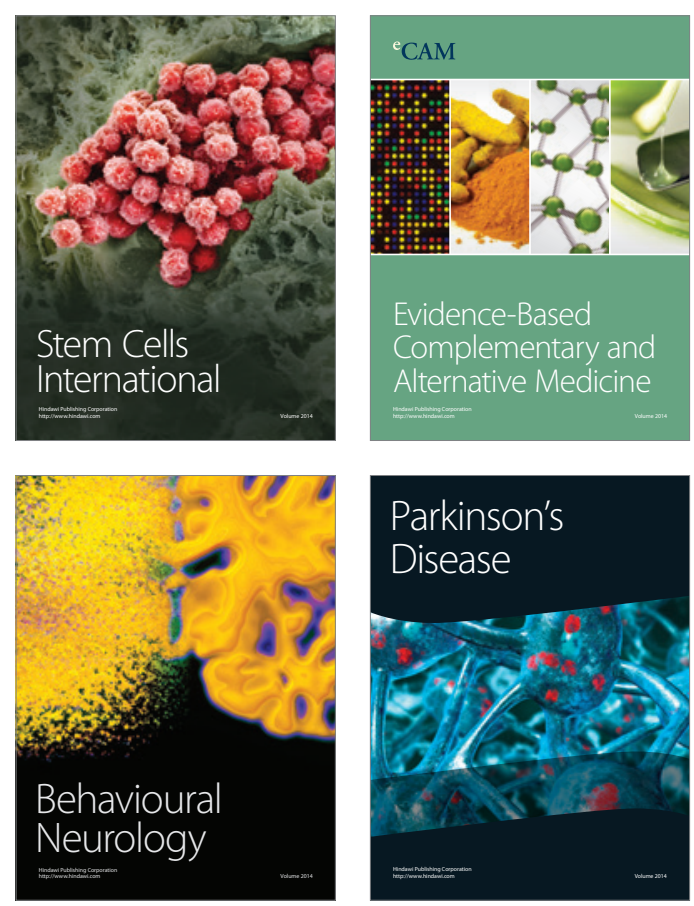

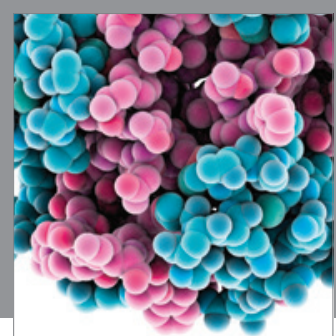

Journal of
Diabetes Research

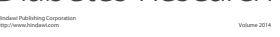

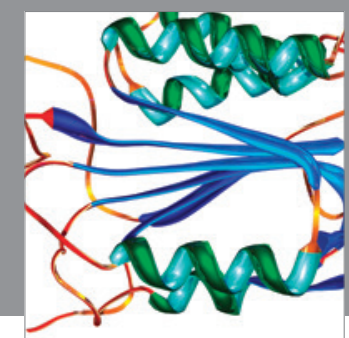

Disease Markers
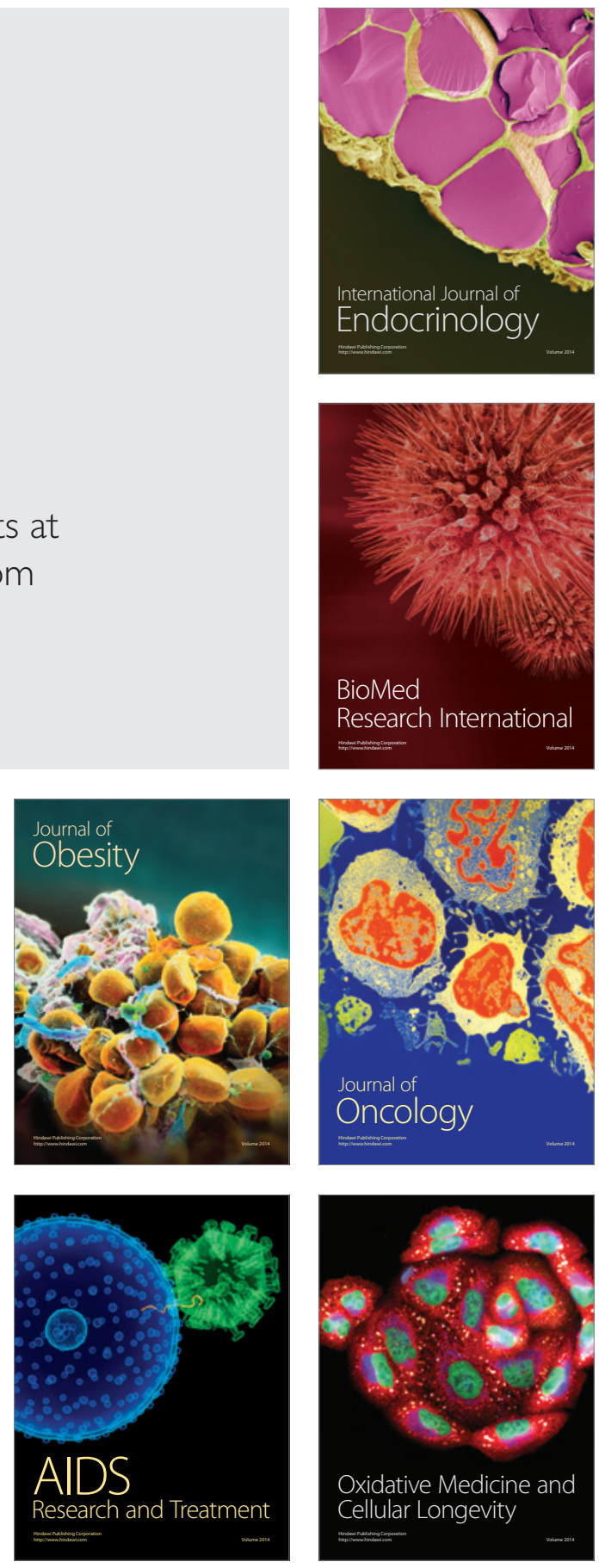